\title{
Survey of PDA management in very low birth weight infants across Italy
}

\author{
Benjamim Ficial ${ }^{{ }^{*+}}$ (D) luri Corsini ${ }^{2+}$, Stefano Fiocchi ${ }^{3+}$, Federico Schena ${ }^{4+}$, Irma Capolupo ${ }^{5}$, Rosa Maria Cerbo ${ }^{6}$, \\ Manuela Condò ${ }^{7}$, Daniela Doni ${ }^{8}$, Simona La Placa ${ }^{9}$, Salvatore Porzio ${ }^{10}$, Katia Rossi ${ }^{11}$, Sabrina Salvadori ${ }^{12}$, \\ Marilena Savoia ${ }^{13}$ and On the behalf of the Italian Study Group of Neonatal Cardiology of the Italian Society of \\ Neonatology
}

\begin{abstract}
Background: The optimal management of PDA in very low birth weight (VLBW) infants is still controversial. Aim of our study was to investigate the management of PDA in the Italian neonatal intensive care units (NICU).

Methods: We conducted an on-line survey study from June to September 2017. A 50-items questionnaire was developed by the Italian Neonatal Cardiology Study Group and was sent to Italian NICUs.

Results: The overall response rate was 72\%. Diagnosis of PDA was done by neonatologists, cardiologists or both (62, 12 and 28\% respectively). PDA significance was assessed by a comprehensive approach in all centers, although we found a heterogeneous combination of parameters and cut-offs used. None used prophylactic treatment. 19\% of centers treated PDA in the first $24 \mathrm{~h}, 60 \%$ after the first $24 \mathrm{~h}$, following screening echocardiography or clinical symptoms, $18 \%$ after the first $72 \mathrm{~h}$ and $2 \%$ after the first week. In the first course of treatment ibuprofen, indomethacin and paracetamol were used in 87,6 and $7 \%$ of centers respectively. Median of surgical ligation was $3 \%(1-6 \%)$.

Conclusions: Significant variations exist in the management of PDA in Italy. Conservative strategy and targeted treatment to infants older than $24 \mathrm{~h}$ with echocardiographic signs of hemodynamic significance seemed to be the most adopted approach.
\end{abstract}

Keywords: Survey, Preterm infants, PDA management, Neonatologist performed echocardiography

\section{Backgrounds}

Despite decades of research, there is still controversy on the optimal management of patent ductus arteriosus (PDA) in very low birth weight (VLBW) infants [1]. While in term infants PDA usually closes within $72 \mathrm{~h}$ of life, in preterm closure is delayed, allowing blood to flow from the aorta to the pulmonary arteries, leading to systemic under-perfusion and pulmonary over-circulation. The magnitude of the left to right shunt is responsible of the hemodynamic consequences of PDA and it is associated to various clinical problems: pulmonary hemorrhage,

\footnotetext{
* Correspondence: benjamim.ficial@gmail.com

${ }^{\dagger}$ Benjamim Ficial, luri Corsini, Stefano Fiocchi and Federico Schena

contributed equally to this work. Marilena Savoia - Chair of the Italian Study

Group of Neonatal Cardiology.

${ }^{1}$ Neonatal Intensive Care Unit, Azienda Ospedaliera Universitaria Integrata di Verona, P.le Stefani 1, 37126 Verona, Italy

Full list of author information is available at the end of the article
}

hypotension, intraventricular hemorrhage (IVH), bronchopulmonary dysplasia (BPD), necrotizing enterocolitis (NEC), mortality $[2,3]$.

In the past, to reduce the burden of these comorbidities, early routine closure of PDA was the preferred approach but did not improve long-term outcomes [4]. Fifty randomized controlled trials (RCTs) enrolling 4878 infants showed that early routine closure of PDA has no effect on the most important outcomes (BPD, NEC, neurodevelopmental impairment) with narrow confidence intervals [5].

In the last ten years, the lack of demonstrable improvement following non selective PDA treatment paved the way to a more conservative approach, that led to lower rates of medical and surgical closure, without compromising long-term outcomes [6].

On the other side, the increasing utilization of echocardiography by neonatologist, namely Neonatologist 
Performed Echocardiography (NPE), allowed targeting treatment to neonates with high/moderate ductal shunt, those were likely to benefit from PDA closure [7]. There is emerging evidence that prolonged exposure to high/ moderate ductal shunt is associated with BPD and that early pharmacological treatment might reduce the incidence of BPD, although there is still no robust evidence from RCTs that treating these high-risk patients improves outcome $[8,9]$

Currently, there is no clear evidence to help neonatologists identify which patient to treat, if any, when and with which drug, therefore the approach to both diagnosis and treatment of PDA has huge variations within individual centers, between institutions and countries [10]. Data on the current management of PDA were previously reported, but, to the best of our knowledge, no data were available from Italy [11-13].

Aim of our study was to investigate the management of PDA in VLBW infants across the Italian NICUs.

\section{Methods}

We conducted a prospective cross-sectional survey study from June to September 2017.

\section{Questionnaire}

Four members (I.C., B.F., S.F., F.S.) of the Italian Study Group of Neonatal Cardiology (ISGNC) developed a 50item questionnaire according to the CHERRIES method for internet e-surveys [14]. The questionnaire was divided into 4 sections: diagnosis of a hemodynamically significant PDA (hsPDA), pharmacological, nonpharmacological and surgical management. All questions were loaded to the Google Forms Website, a free tool for creating online survey forms (https://docs.google.com/ forms $/ \mathrm{u} / 0 /$ ) and were proofread. Prior to distribution, the survey was pilot-tested to identify potential inaccuracies by the other 9 members of the ISGNC and modified accordingly, as previously reported [15]. The questionnaire required approximately $30 \mathrm{~min}$ to be completed.

\section{Web-based survey}

A cover letter containing a hyperlink to the survey was sent to the directors of the Italian Neonatal Units and to one attending neonatologist at each site with expertise in neonatal cardiology, based on the database of the Italian Society of Neonatology and the ISGNC. To ensure only one response for center an unique survey link was assigned to each institution. To prevent incomplete answers, the survey form could be submitted only when completed.

The first email was sent in June 2017 and a reminder in September 2017. No responders were subsequently contacted by a personalized e-mail and/or a phone call. No financial rewards were offered for taking part in the survey. Completion of the questionnaire implied consent to take part in the survey. The identity of each participant was kept confidential throughout the data collection and analysis. The missing responses of some centres defined the study as 'voluntary inquiry with presence of non-respondents'.

\section{Data analysis}

Google Forms automatically converted every questionnaire into Excel files (Microsoft, Seattle, WA). I.C. and B. F checked every questionnaire for possible inconsistencies throughout this process of conversion. Continuous variables were tested for normality using the Shapiro-Wilk test and presented as means (SD) or median (IQR) as appropriate. Categorical variables were presented as proportions. Comparisons between subgroups were conducted using a Student $t$ test or a Mann-Whitney $U$ test as appropriate. Categorical variables were compared using the $\chi^{2}$ or Fisher exact test as appropriate. A $p$ value of $<0.05$ was considered significant. SPSS v 20 (SPSS Inc., Chicago, Illinois) was used to perform the statistical analysis.

\section{Results}

\section{Epidemiological and organisational characteristics of the} centers

The overall survey response rate was $72 \%(82 / 114)$. Among respondent centers, $85 \%$ were classified as level III and $15 \%$ as level IV neonatal units [16]. Comparing answers of early versus late respondents (i.e. after phone call reminder), we did not find statistically significant differences. In Fig. 1 the centers are divided according to the number of VLBW per year.

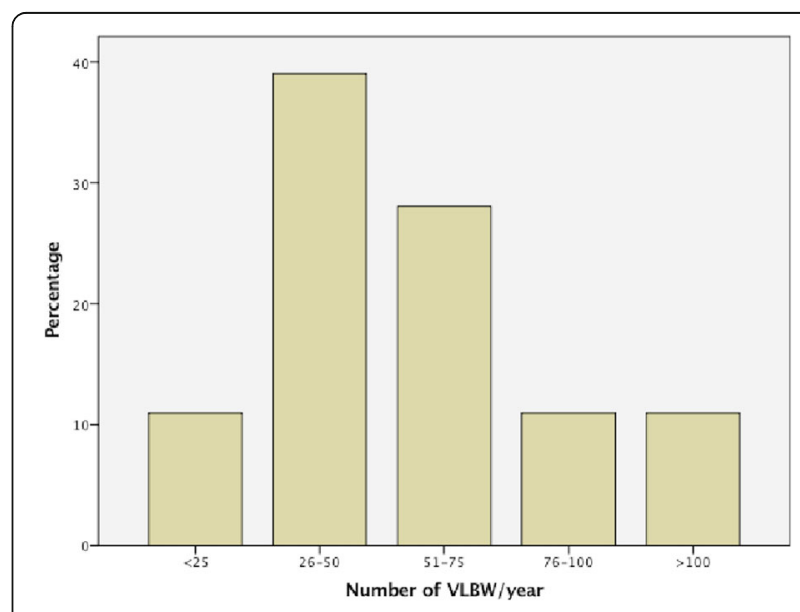

Fig. 1 Italian NICUs divided according to the volume of activity, defined as the number of very low birth weight (VLBW) infants per year 


\section{Diagnosis of PDA}

In $68 \%$ of centers there was an institutional protocol for PDA management. Neonatologist, cardiologist or both usually performed the echocardiography to assess a PDA in a VLBW neonate in 60,12 and $28 \%$ of centers respectively. The first echocardiogram was performed in the first $24 \mathrm{~h}$ of life, between the 24 and $72 \mathrm{~h}$ or after the first $72 \mathrm{~h}$ in the 31, 60 and $9 \%$ of centers respectively.

In $87 \%$ of centers the first echocardiogram was always a complete structural examination to exclude the presence of CHD. In the remaining cases, a complete examination was performed only if CHD was suspected (7\%) or before starting treatment $(6 \%)$. Neonatologist, cardiologist or both excluded CHDs in the 51, 27 and 22\% respectively.

Figure 2 shows all the echocardiographic parameters used for the assessment of PDA and their percentage of use.

PDA diameter was measured at its narrowest point at the pulmonary end from a 2D image, from a color Doppler flow image or both in 24, 20 and 56\% respectively.

In $74 \%$ of centers, the absolute value of PDA diameter was used. The PDA diameter was indexed to patient weight and to body surface area in 24 and $1 \%$ of centers respectively.

Median cut-off value for hsPDA diameter was $1.5 \mathrm{~mm}$ $(1.5-2 \mathrm{~mm})$ and for PDA diameter indexed to body weight was $1.4 \mathrm{~mm} / \mathrm{kg}(1.4-1.5 \mathrm{~mm} / \mathrm{kg})$.

Flow across a PDA was assessed as follows: peak systolic flow velocity in the $57 \%$ of centers, end diastolic flow velocity in the $16 \%$, ratio between peak systolic and end diastolic flow velocities in the $12 \%$, type of flow pattern across a PDA in the $99 \%$.

Pulmonary over-circulation was assessed as follows: left atrium/aortic root ratio (LA/Ao) in 95\% of centers, ratio between $\mathrm{E}$ and $\mathrm{A}$ wave of transmitral flow $(\mathrm{E} / \mathrm{A})$ in $18 \%$, left ventricular output (LVO) in 37\%, end-diastolic flow velocity of left pulmonary artery (LPA) in $43 \%$, left ventricle isovolumic relaxation time (IVRT) in $1 \%$.

Among centers that used LA/Ao, median cut-off value of hsPDA was 1.5 (1.4-1.5).

Systemic hypo-perfusion was assessed as follows: descending aortic flow pattern in $79 \%$ of centers, flow pattern in peripheral vessels in $83 \%, \mathrm{LVO} / \mathrm{SVC}$ flow ratio in $22 \%$.

The peripheral vessels examined were cerebral, celiac or mesenteric and renal in the 90, 69 and 31\% respectively.

Calculation of resistance index, qualitative evaluation of flow pattern or both were used in the 31, 17 and 52\% respectively. Table 1 compares the percentage of use of echocardiographic parameters by neonatologists and cardiologists.

Only in $8 \%$ of centers the echocardiographic assessment of PDA satisfied the requirements proposed by the recently released indications of the "European Special Interest Group Neonatologist Performed Echocardiography" [17].

Near infrared spectroscopy (NIRS) was used in the $25 \%$ of centers: cerebral district alone was evaluated in the $6 \%$ of centers whereas both cerebral and renal district were evaluated in the $19 \%$.

Most centers (84\%) did not use biochemical markers when assessing a PDA, 13 and 3\% of centers used brain natriuretic peptide (BNP) and troponin respectively.

\section{Medical treatment}

Medical treatment of hsPDA was decided by pediatric cardiologist, neonatologist or neonatologist in collaboration with cardiologist in 3, 57 and $40 \%$ of centers respectively.

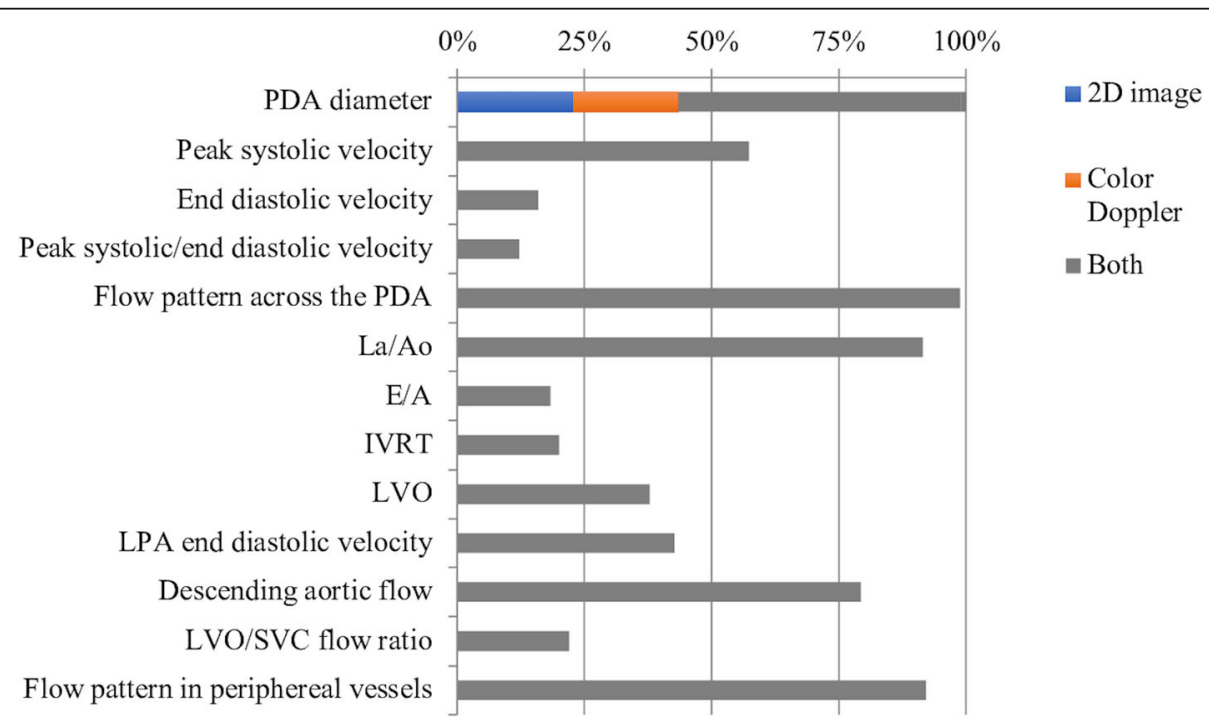

Fig. 2 Echocardiographic parameters used among Italian NICUs for the assessment of PDA and their percentage of use. LA Left Atrium, Ao Aorta, E Mitral E wave, A Mitral A wave, IVRT Isovolumic relaxation time, LVO Left ventricular output, LPA Left pulmonary artery, SVC Superior vena cava 
Table 1 Comparison between echocardiographic parameters used by neonatologist and cardiologist in PDA assessment. E mitral E wave, A mitral A wave, LA/Ao Left Atrium Aortic root ratio, IVRT Isovolumic relaxation time, LVO Left ventricular output, LPA Left pulmonary artery, SVC superior vena cava

\begin{tabular}{llll}
\hline Echocardiographic parameter & Echo assessment by neonatologist & Echo assessment by cardiologist \\
\hline PDA diameter & $100 \%$ & $100 \%$ \\
Ductal Peak systolic velocity & $54 \%$ & $80 \%$ \\
Ductal End diastolic velocity & $14 \%$ & $30 \%$ \\
Ductal Peak systolic/End diastolic velocity & $13 \%$ & $10 \%$ \\
Flow pattern across PDA & $100 \%$ & $90 \%$ \\
LAVAo & $95 \%$ & $100 \%$ \\
E/A & $19 \%$ & $10 \%$ \\
IVRT & $1 \%$ & $0 \%$ \\
LVO & $40 \%$ & $20 \%$ \\
LPA end diastolic velocity & $44 \%$ & $30 \%$ \\
Descending aortic flow & $79 \%$ & $80 \%$ \\
LVO/SVC flow ratio & $22 \%$ & $20 \%$ \\
Flow pattern in peripheral vessels & $89 \%$ & $40 \%$ \\
\hline
\end{tabular}

$*=p<0.05$

Median incidence of medical treatment was $22 \%$ $(17-30 \%)$.

Prophylactic treatment was not adopted among Italian NICUs. Figure 3 shows timing and treatment strategies of medical treatment of PDA among Italian NICUs. The timing of the first course of medical treatment showed marked variability. Nineteen per cent of centers started PDA treatment in the first day of life (13\% based on presymptomatic echocardiographic screening, $6 \%$ based on signs and/or symptoms of PDA).

The majority of Italian NICUs did not treat babies in the first $24 \mathrm{~h}$ and considered pharmacological closure of PDA between the first 24 and $72 \mathrm{~h}$ of life $(60 \%)$,

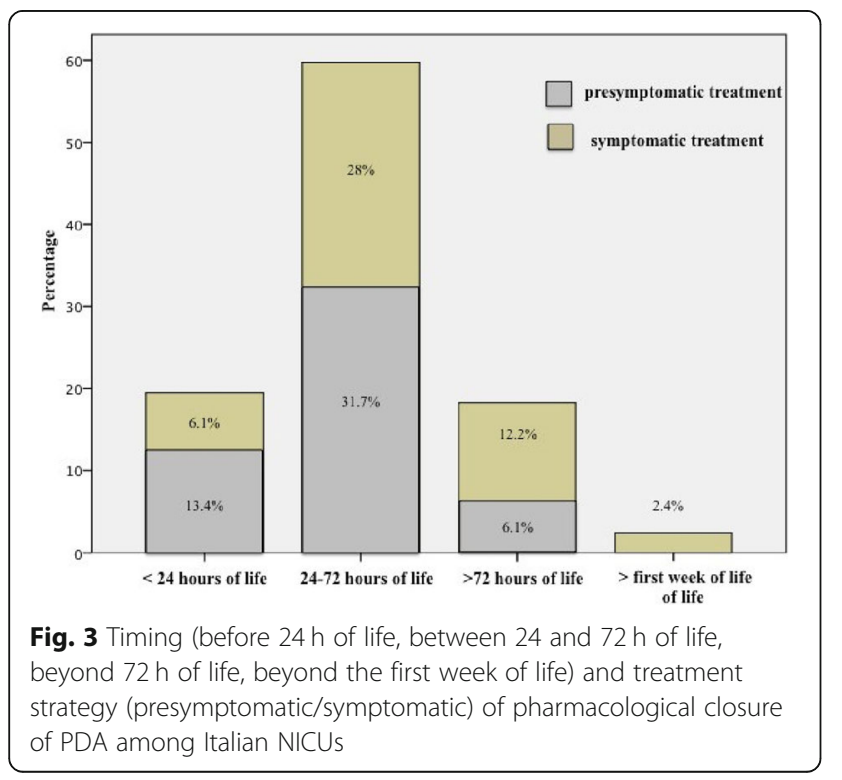

adopting both a pre-symptomatic and symptomatic treatment (32 and 28\% respectively). Eighteen percent of centers considered treatment of PDA only after the first $72 \mathrm{~h}$ of life ( $12 \%$ symptomatic and $6 \%$ presymptomatic) and $2 \%$ of centers did not treat PDA in the first week of life.

Comparing frequency of medical treatment in centers that adopted pre-symptomatic and symptomatic treatment we did not found significant differences (median $22 \%$ for both groups).

In the first course ibuprofen, indomethacin and paracetamol were used in 87, 6 and $7 \%$ of centers respectively.

Ibuprofen was administered in bolus, continuous infusion and orally in the 91,5 and $4 \%$ respectively. The most common ibuprofen regimen was $10 \mathrm{mg} / \mathrm{kg}$ for the first dose followed by $5 \mathrm{mg} / \mathrm{kg}$ for the remaining two doses $(92 \%)$. In $5 \%$ of centers ibuprofen $20 \mathrm{mg} / \mathrm{kg}$ for the first dose, followed by $10 \mathrm{mg} / \mathrm{kg}$ was used. In $2 \%$ both low and high dose ibuprofen regimens were used. The majority of the units (73\%) used a 3 days course, instead $27 \%$ of units stopped the course earlier if PDA closed at daily follow-up echos.

Indomethacin was given by intravenous bolus infusion. In the majority of centers (95\%) the first dose was 0.2 $\mathrm{mg} / \mathrm{kg}$ and the following doses ranged from 0.1 to 0.25 $\mathrm{mg} / \mathrm{kg}$ according to the day of life $(0.1 \mathrm{mg}$ in the first 48 $\mathrm{h}, 0.2 \mathrm{mg} / \mathrm{kg}$ from day 3rd to 7 th of life, $0.25 \mathrm{mg} / \mathrm{kg}$ after the first week). The duration was three or six doses (57 and $3 \%$ respectively). Forty percent of centers stopped the course earlier if PDA closed.

Paracetamol was given orally, intravenously (bolus infusion) or both in the 68,18 and $14 \%$ respectively. The 
most common dose regimen was $15 \mathrm{mg} / \mathrm{kg}$ four times a day (92\%), followed by $10 \mathrm{mg} / \mathrm{kg}$ and $7.5 \mathrm{mg} / \mathrm{kg}$ four times a day ( 6 and $2 \%$ respectively). The duration of the cycle of paracetamol was 3 or 6 days in the 49 and $24 \%$ respectively. Twenty-seven percent of centers stopped the course earlier if PDA closed.

If the first course failed to close the duct, a second course with ibuprofen, indomethacin or paracetamol was used in $67,14,11 \%$ respectively. In $3 \%$ of centers patients underwent to surgical ligation and in the remaining 5\% "watchful waiting".

Among centers that used a second course of ibuprofen, the dose regimen was $10 \mathrm{mg} / \mathrm{kg}$ for the first dose and $5 \mathrm{mg} / \mathrm{kg}$ for two doses in the $74 \%$ of cases and 20 $\mathrm{mg} / \mathrm{kg}$ for the first dose and $10 \mathrm{mg} / \mathrm{kg}$ for two doses in the $26 \%$.

If the second course failed, the management options were: surgical ligation in $46 \%$, a third course with ibuprofen $(10 \%)$, indomethacin $(23 \%)$ or paracetamol (15\%), "watchful waiting" (6\%).

The most frequent contraindications of ibuprofen and indomethacin were: diuresis $<0.5 \mathrm{~m} / \mathrm{kg} / \mathrm{h}(41 \%)$, diuresis $<1 \mathrm{ml} / \mathrm{kg} / \mathrm{h}$ (47\%), Platelets $<50.000 / \mu \mathrm{L}$ (77\%), Platelets $<30.000 / \mu \mathrm{L}(17 \%)$, sepsis $(30 \%), \mathrm{IVH} \geq$ grade $3(90 \%)$, NEC (80\%), increased creatinine (6\%), every grade IVH (2\%).

To treat PDA in babies with severe IVH or NEC, paracetamol, surgical ligation or conservative management followed by course with ibuprofen were adopted in 44, 33 and $23 \%$ of centers respectively.

\section{Supportive therapy}

Fluid restriction was used in all infants with PDA in 47\% of centers and only in infants with hsPDA in $43 \%$. In $10 \%$ of centers fluid restriction was not used.

Enteral feeding was continued, reduced or totally withdrawn during pharmacological closure of PDA in 56, 37 and $7 \%$ of centers respectively.

Seventy per cent of centers did not used gastroprotective drugs during treatment with NSAIDs, 26 and $5 \%$ used ranitidine and omeprazole respectively.

Thirty-three per cent of centers used increased PEEP values for neonates with hsPDA.

Treatment of congestive heart failure varied between centers: standard dose furosemide $(1-2 \mathrm{mg} / \mathrm{kg}$ ) was used in $81 \%$ of centers, high dose furosemide $(>2 \mathrm{mg} / \mathrm{kg})$ in $6 \%$, fluid restriction in $67 \%$, digoxin in $3 \%$, dobutamine in $1 \%$, hydrochlorothiazide and spironolactone in $6 \%$.

\section{Surgical treatment}

Surgical ligation was decided by pediatric cardiologist, neonatologist or neonatologist in collaboration with cardiologist in 7, 44 and $49 \%$ of centers respectively. Median incidence of surgical ligation was 3\% (1-6\%).
Surgical ligation was performed by cardiac surgeons of the same institution or of other institutions in 18 and $60 \%$ respectively. Surgical candidates were transferred to referral centers in the remaining $22 \%$. In $91 \%$ of centers surgical ligation was performed at the cot-side in the NICU.

Surgical ligation was performed after failure of the first, second or third cycle of medical therapy in 3, 46 and $44 \%$ of centers respectively. In the remaining $7 \%$ of centers surgical ligation was not considered. Thirtythree per cent of centers performed surgical ligation immediately without trying medical therapy in babies with NEC or severe IVH. After surgery $79 \%$ of centers performed echocardiographic screening for post-ligation cardiac syndrome.

\section{Discussion}

We investigated the PDA management in Italian neonatal units, the survey received a high response rate, that, although not optimal, was comparable to previous similar reports $[18,19]$. Although the suboptimal response rate may be considered a limitation of the current survey, studies of non-response to pediatric surveys found that lower response rates were not necessarily related to bias [18]. Moreover, we found a low nonresponse bias comparing early versus late respondents. Therefore, we may conclude that our results were representative of the current clinical practice in Italy.

We found a great variability in diagnosis, medical, surgical and supportive treatment, as previously reported in other countries [ 11-13, 15]. Lack of evidence-based guidelines for the management of PDA was likely to be a major contributor to this variability [3]. Our survey was sent to a single neonatologist at each site with expertise in neonatal cardiology, we did not directly investigated intra-institutional practice variations. We speculate that major differences were likely to exist also within the same unit, only $68 \%$ of centers having institutional protocol for PDA management in place, similarly to other countries $[10,11,15]$.

\section{Variability in approach to diagnosis}

An ECHO-based approach for diagnosing ductal patency and assessing hemodynamic significance was widely adopted in Italian NICUs, according to the current evidence that proposes echocardiography as the most clinically applicable modality $[1,18]$. The homogeneous implementation of an ECHO-based approach is likely to have different reasons. First, the vast majority of Italian NICUs had access to NPE, as shown from our previous survey: NPE was performed in $95 \%$ of centers and in $70 \%$ of centers on a $24 / 7$ basis [20]. Secondly, clinical signs of PDA take 2 to 3 days to appear and have poor sensitivity and specificity [21]. Finally, 
early comprehensive echocardiography is able to assess shunt volume entity, possibly identifying neonates at higher risk of PDA comorbidities [22, 23].

Italian NICUs consistently used comprehensive echocardiography to assess shunt volume rather than an approach based on single parameter (i.e. ductal diameter), allowing for a more accurate evaluation of shunt entity. Reliance only on PDA size has been recently put in doubt, as the vast majority of RCTs that based interventions only on ductal diameter failed to show any improvements in PDA related morbidities [24] Many combinations of ultrasound parameters were proposed as surrogates of shunt volume to define a hsPDA, nevertheless robust agreement is proving difficult among neonatologists [25].

This was confirmed by our data: we found a heterogeneous combination of parameters meant to assess PDA. The combination more frequently adopted in Italy was similar to the one reported by a French survey [11]. No significant differences were found comparing cardiologist and neonatologist approach, except for the evaluation of flow reversal in peripheral vessels.

Following survey completion, a consensus statement of ESPR/ESN has been released aiming to standardize the echocardiographic assessment of hemodynamic significance of a PDA in terms of parameters used and cut-offs [17]. A minority of our centers met these essentials requirements, in particular, LVO, that is considered an essential index of pulmonary over-circulation, was adopted by a minority of centers and needs to be implemented.

Similarly to other European countries, Australia and New Zealand, echocardiographic assessment of PDA was more frequently performed by neonatologists rather than cardiologists [10-13].

In a minority of cases, the first evaluation was not a comprehensive study aimed to confirm normal cardiac anatomy, as recommended by the recently released ESPR/ESN consensus statement [26, 27]. Although in the remaining cases normal heart structure was confirmed afterwards, this is a major concern of the NPE assessment of PDA due to the risk of misdiagnosing critical CHD [26].

Considering the number of serial echo assessments required in preterm neonates, the need to integrate the ultrasound findings with clinical data and the limited access to pediatric cardiology service in Italy, echocardiographic assessment of PDA should probably remain the domain of a properly trained neonatologist, in collaboration with cardiologist whenever needed, to minimize the risk of misdiagnosis of critical CHDs [20]. In order to meet training needs and to pursue quality assurance and patient safety, our INCSG is currently designing and implementing a formalised and accredited training program in close collaboration with the Italian Society of Pediatric
Cardiology (SICPED). This will be of major help to uniform and standardize the echocardiographic PDA assessment among neonatal units in Italy.

\section{Variability in approach to medical treatment}

Approach to medical treatment of PDA varied markedly among Italian NICUs, yet we found an overall tendency towards a conservative management, in which pharmacotherapy and/or surgical ligation decreased, similarly to the data recently reported by other countries (USA, Canada) $[28,29]$. Data reported by the Italian Neonatal Network (Vermont Oxford Network) for the year 2017 confirmed our results, despite Survey method was not the optimal tool to assess the incidence of medical treatment of PDA among Italian NICUs.

Early medical treatment between 24 and $72 \mathrm{~h}$ of life seemed to be the most adopted strategy. This approach is supported by recent data showing that treating babies with high to moderate shunt volume across the PDA in the first 2-3 days of life might reduce BPD. Apparently, a critical window may be present during the first seven to ten days of life, in which exposure to a high-moderate shunt through PDA may increase the risk of BPD and pulmonary comorbidities. After this period, PDA treatment does not seem to influence BPD development [9]. The majority of Italian centers adopted a presymptomatic approach, performing early screening echocardiography before the onset of PDA clinical signs. This approach is based on the rationale that treating neonates at a preclinical stage might avoid PDA comorbidities. Short and long-term benefits of this strategy remain to be proven, despite some emerging data would show less pulmonary hemorrhage, in-hospital mortality and BPD compared to controls [7, 30]. Pre-symptomatic approach was less common in other European countries (UK and France), compared to Italy $[11,13]$. These data were retrieved some years ago, recent advancements in NPE for PDA assessment may have led neonatologists to a change from previous practice [21].

Prophylactic indomethacin was not used: similarly to other countries, this approach, that gained popularity in the ' 90 s, is declining, possibly because subsequent data did not find long-term improvements [4].

In the vast majority of centers ibuprofen was the drug of choice in the first course of medical therapy, whereas a minority of centers used indomethacin and paracetamol. Use of ibuprofen as first line drug was consistent with other European countries, considering that it carries decreased risk of renal impairment and similar efficacy [31].

Standard dosage regimen of ibuprofen was universally adopted by almost all Italian NICUs [32]. A minority of centers used the high dose regimen, in particular when the first course failed to close the duct [33]. 
Indomethacin use increased after first course of ibuprofen failed, possibly because indomethacin effectiveness is not affected by late treatment [34]. Short course and low dosing regimen were more commonly used, most likely because equally effective in ductal closure and inducing less renal impairment $[35,36]$.

We found a significant use of paracetamol, both as first line and as rescue therapy. There is mounting evidence of its efficacy, particularly where NSAIDs are contraindicated or unsuccessful [37].

Severe IVH and NEC were common contraindications to NSAIDs, as well as oliguria and thrombocytopenia, with yet different cut-offs.

\section{Variability in approach to supportive treatment}

Despite conflicting evidence, conservative interventions have been increasingly adopted as a therapeutic modality in recent years $[38,39]$. The lack of standardization of this practice was confirmed by our data: no standard criteria and modalities for fluid restriction, diuretics and endexpiratory pressure were adopted among Italian centers.

\section{Variability in approach to surgical ligation}

Due to concerns about adverse neonatal outcomes, in the last decade surgical ligation rate decreased. Our data confirmed this tendency in Italian NICUs. In the vast majority of centers surgical ligation was performed at the NICU bedside by an on-site or an off-site team, to eliminate the risks associated with transferring instable babies to referring hospitals, according to the recent evidence [40].

\section{Conclusions}

Approach to both diagnosis and treatment of PDA among Italian NICUs was highly variable and it would be interesting to confirm this variability in the management of PDA in a European perspective. No homogeneous criteria for PDA diagnosis were found, although an ECHO-based approach with a comprehensive echocardiographic assessment was widely adopted.

Conservative strategy and targeted treatment to infants older than $24 \mathrm{~h}$ with echocardiographic signs of moderate to high left to right shunt volume across the PDA seemed to be the most adopted approach. While awaiting further evidence on PDA management from ongoing trials, our data confirmed the need of standardization of clinical practice, which would hopefully improve clinical management and trials methodology and results.

\section{Supplementary information}

Supplementary information accompanies this paper at https://doi.org/10. 1186/s13052-020-0773-0.

Additional file 1. List of Neonatal Units respondent to the survey.

\section{Abbreviations}

A: Mitral A wave; BNP: Brain natriuretic peptide; BPD: Bronchopulmonary dysplasia; CHD: Congenital heart disease; E: Mitral E wave; ESN : European Society of Neonatology; ESPR : European Society of Pediatric Research; hsPDA: Hemodynamically significant PDA; IQR: Interquartile range; ISGNC: Italian Study Group of Neonatal Cardiology; IVH: Intraventricular hemorrhage; IVRT: Isovolumic relaxation time; LA/Ao: left atrium/aortic root ratio; LPA: Left pulmonary artery; LVO: Left ventricular output;

NEC: Necrotizing enterocolitis; NICU: Neonatal intensive care unit; NIRS: Near infrared spectroscopy; NPE: Neonatologist performed echocardiography; NSAID: Nonsteroidal anti-inflammatory drug; PDA: Patent ductus arteriosus; RCT: Randomized controlled trial; SD: Standard deviation; ISPC: Italian Society of Pediatric Cardiology; SVC: Superior vena cava; VLBW: Very low birth weight

\section{Acknowledgements}

The authors would like to thank our colleagues from all Italian NICUs that responded to the survey (see list of neonatal units in Additional file 1).

\section{Authors' contributions}

$\mathrm{BF}, \mathrm{IC}, \mathrm{SF}$ and FS designed the study, contributed to acquisition, analysis and interpretation of data, drafted the article. IaC, RMC, MC, DD, SL, SP, KR, SS contributed to acquisition of data and revised the article. MS contributed to analysis and interpretation of data and revised the article. All authors approved the final version of the article.

\section{Funding}

This work was supported by Orphan-Europe Italy. Study sponsor had no involvement in data collection, analysis or interpretation.

\section{Availability of data and materials}

The data that support the findings of this study are available from Italian Society of Neonatology but restrictions apply to the availability of these data, which were used under license for the current study, and so are not publicly available. Data are however available from the corresponding author upon reasonable request and with permission of Italian Society of Neonatology.

Ethics approval and consent to participate

Not applicable.

\section{Consent for publication}

Informed consent was obtained from all individual participants included in the study.

\section{Competing interests}

The authors declare that they do not have any financial relationships that could be relevant to this work. The authors declare no conflicts of interest in relation to the manuscript.

\section{Author details}

${ }^{1}$ Neonatal Intensive Care Unit, Azienda Ospedaliera Universitaria Integrata di Verona, P.le Stefani 1, 37126 Verona, Italy. ${ }^{2}$ Division of Neonatalogy, Careggi University Hospital of Florence, Florence, Italy. ${ }^{3}$ Neonatologia e Terapia Intensiva Neonatale, Ospedale Valduce, Como, Italy. ${ }^{4}$ Neonatal Intensive Care Unit, Fondazione IRCCS Cà Granda Ospedale Maggiore Policlinico di Milano, Milan, Italy. ${ }^{5}$ Neonatal Intensive Care Unit, Ospedale Pediatrico Bambino Gesù, Rome, Italy. ${ }^{6}$ Neonatal Intensive Care Unit, Fondazione IRCCS Policlinico San Matteo, Pavia, Italy. ${ }^{7}$ Neonatal Intensive Care Unit, Ospedale A. Manzoni, Lecco, Italy. ${ }^{8}$ Neonatal Intensive Care Unit, FMBBM San Gerardo, Monza, Italy. ${ }^{9}$ Neonatal Intensive Care Unit, AOUP Giaccone, Palermo, Italy. ${ }^{10}$ Neonatal Section, San Michele Hospital, Maddaloni, NA, Italy. ${ }^{11}$ Neonatal Intensive Care Unit, Policlinico di Modena, Modena, Italy. ${ }^{12}$ Neonatal Intensive Care Unit, Azienda Ospedaliera-Università di Padova, Padova, Italy.

${ }^{13}$ Neonatal Intensive Care Unit, Azienda Ospedaliera Universitaria S Maria della Misericordia, Udine, Italy.

Received: 8 October 2019 Accepted: 12 January 2020

Published online: 14 February 2020

\section{References}

1. Jain A, Shah PS. Diagnosis, evaluation and Management of Patent Ductus Arteriosus in preterm neonates. JAMA Pediatr. 2015;169(9):863-72. 
2. Hamrick SE, Hansmann G. Patent ductus arteriosus of the preterm infant Pediatrics. 2010;125(5):1020-30.

3. McNamara PJ, Sehgal A. Towards rational management of the patent ductus arteriosus: the need for disease staging. Arch Dis Child Fetal Neonatal Ed. 2007;92(6):F424-7.

4. Benitz WE and COMMITTEE ON FETUS AND NEWBORN. Patent Ductus Arteriosus in preterm infants. Pediatrics 2016;137(1):e20153730.

5. Benitz WE. Treatment of persistent patent ductus arteriosus in preterm infants: time to accept the null hypothesis? J Perinatol. 2010;30(4):241-52.

6. EL-Khuffash A, Weisz DE, McNamara PJ. Reflections of the changes in patent ductus arteriosus management during the last 10 years. Arch Dis Child Fetal Neonatal Ed 2016;101(5):F474-F478.

7. Rozé JC, Cambonie G, Marchand-Martin L, Gournay V, Durrmeyer X, Durox $M$, Storme L, et al. Hemodynamic EPIPAGE 2 study group. Association between early screening for patent Ductus Arteriosus and in-hospital mortality among extremely preterm infants. JAMA. 2015;313(24):2441-8.

8. Schena F, Francescato G, Cappelleri A, Picciolli I, Mayer A, Mosca F, et al. Association between Hemodynamically significant patent Ductus Arteriosus and Bronchopulmonary dysplasia. J Pediatr. 2015;166(6):1488-92.

9. Clyman RI. Patent ductus arteriosus, its treatments, and the risks of pulmonary morbidity. Semin Perinatol. 2018;42(4):235-42.

10. Sehgal A, McNamara PJ. International perspective on management of a patent ductus arteriosus: lessons learned. Semin Fetal Neonatal Med. 2018, 23(4):278-84.

11. Brissaud O, Guichoux J. Patent Ductus Arteriosus in the preterm infant: A survey of clinical practices in French neonatal intensive care units. Pediatr Cardiol. 2011;32:607-14.

12. Hoellering $A B$, Cooke $L$. The management of patent ductus arteriosus in Australia and New Zealand. J Paediatr Child Health. 2009:45(4):204-9.

13. Kulkarni A, Richards J, Duffy D. Survey of management of patent ductus arteriosus in neonatal units across England. Arch Dis Child Fetal Neonatal Ed. 2013;98:F465-6.

14. Eysenbach G. Improving the quality of web surveys: the checklist for reporting results of internet E-surveys (CHERRIES). J Med Internet Res. 2004;6:1-6.

15. Amin SB, Handley C, Carter-Pokras O. Indomethacin use for the management of patent ductus arteriosus in preterms: a web-based survey of practice attitudes among neonatal fellowship program directors in the United States. Pediatr Cardiol. 2007;28(3):193-200.

16. COMMITTEE ON FETUS AND NEWBORN. Levels of Neonatal Care Pediatrics 2012;130;587.

17. van Laere D, van Overmeire B, Gupta S, El Khuffash A, Savoia M, McNamara PJ, et al. European special interest group "neonatologist performed echocardiography" (NPE). Application of NPE in the assessment of a patent ductus arteriosus. Pediatr Res. 2018;84(Suppl 1):46-56.

18. Cull WL, O'Connor KG, Sharp S, Tang SF. Response rates and response bias for 50 surveys of paediatricians. Health Serv Res. 2005;40(1):213-26.

19. Slaughter UL, Reagan PB, Bapat RV, Newman TB, Klebanoff MA. Nonsteroidal anti-inflammatory administration and patent ductus arteriosus ligation, a survey of practice preferences at US children's hospitals. Eur J Pediatr. 2016; 175(6):775-83.

20. Corsini I, Ficial B, Fiocchi S, Schena F, Capolupo I, Cerbo RM, et al. Neonatologist performed echocardiography (NPE) in Italian neonatal intensive care units: a national survey. Ital J Pediatr. 2019;45(1):131.

21. Alagarsamy S, Chhabra M, Gudavalli M, Nadroo AM, Sutija VG, Yugrakh D. Comparison of clinical criteria with echocardiographic findings in diagnosing PDA in preterm infants. J Perinat Med. 2005;33(2):161-4.

22. Sehgal A, Paul E, Menahem S. Functional echocardiography in staging for ductal disease severity: role in predicting outcomes. Eur J Pediatr. 2013; 172(2):179-84

23. El-Khuffash A, James AT, Corcoran JD, Dicker P, Franklin O, Elsayed YN, et al. A Patent Ductus Arteriosus Severity Score Predicts Chronic Lung Disease or Death before Discharge. J Pediatr. 2015;167(6):1354-1361.e2.

24. Shah SS, Ohlsson A. Ibuprofen for the prevention of patent ductus arteriosus in preterm and/or low birth weight infants. Cochrane Database Syst Rev. 2006 Jan 25;1:CD004213.

25. Zonnenberg I, de Waal K. The definition of a haemodynamic significant duct in randomized controlled trials: a systematic literature review. Acta Paediatr. 2012;101:247-51.

26. Groves AM, Singh Y, Dempsey E, Molnar Z, Austin T, El-Khuffash A, et al. European special interest group "neonatologist performed echocardiography" (NPE). Introduction to neonatologist-performed echocardiography. Pediatr Res. 2018 Jul;84(Suppl 1):1-12.

27. de Boode WP, Singh Y, Gupta S, Austin T, Bohlin K, Dempsey E, et al. Recommendations for neonatologist performed echocardiography in Europe: consensus statement endorsed by European Society for Paediatric Research (ESPR) and European Society for Neonatology (ESN). Pediatr Res. 2016;80:465-71.

28. Lokku A, Mirea L, Lee SK, Shah PS. Canadian neonatal network. Canadian neonatal network. Trends and outcomes of patent Ductus Arteriosus treatment in very preterm infants in Canada. Am J Perinatol. 2017;34(5):441-50.

29. Bixler GM, Powers GC, Clark RH, Walker MW, Tolia VN. Changes in the diagnosis and Management of Patent Ductus Arteriosus from 2006 to 2015 in United States neonatal intensive care units. J Pediatr. 2017;189:105-12.

30. Kluckow M, Jeffery M, Gill A, Evans N. A randomised placebo-controlled trial of early treatment of the patent ductus arteriosus. Arch Dis Child Fetal Neonatal Ed;99(2):F99-F104.

31. Thomas RL, Parker GC, Van Overmeire B, Aranda JV. A meta-analysis of ibuprofen versus indomethacin for closure of patent ductus arteriosus. Eur J Pediatr. 2005;164(3):135-40.

32. Aranda JV, Varvarigou A, Beharry K, Bansal R, Bardin C, Modanlou H, et al. Pharmacokinetics and protein binding of intravenous ibuprofen in the premature newborn infant. Acta Paediatr. 1997;86(3):289-93.

33. Desfrere L, Zohar S, Morville P, Brunhes A, Chevret S, Pons G, et al. Dosefinding study of ibuprofen in patent ductus arteriosus using the continual reassessment method. J Clin Pharm Ther. 2005;30(2):121-32.

34. Kaempf JW, Wu YX, Kaempf AJ, Kaempf AM, Wang L, Grunkemeier G. What happens when the patent ductus arteriosus is treated less aggressively in very low birth weight infants? J Perinatol. 2012;32(5):344-8.

35. Jegatheesan P, lanus V, Buchh B, Yoon G, Chorne N, Ewig A, et al. Increased indomethacin dosing for persistent patent ductus arteriosus in preterm infants: a multicenter, randomized, controlled trial. J Pediatr. 2008;153(2):183-9.

36. Herrera C, Holberton J, Davis P. Prolonged versus short course of indomethacin for the treatment of patent ductus arteriosus in preterm infants. Cochrane Database Syst Rev. 2007;2007(2):CD003480.

37. Terrin G, Conte F, Oncel MY, Scipione A, McNamara PJ, Simons S, et al. Paracetamol for the treatment of patent ductus arteriosus in preterm neonates: a systematic review and meta-analysis. Arch Dis Child Fetal Neonatal Ed. 2016;101(2):F127-36.

38. De Buyst J, Rakza T, Pennaforte T, Johansson AB, Storme L. Hemodynamic effects of fluid restriction in preterm infants with significant patent ductus arteriosus. J Pediatr. 2012;161(3):404-8.

39. Hagadorn Jl, Brownell EA, Trzaski JM, Johnson KR, Lainwala S, Campbell BT, et al. Trends and variation in management and outcomes of very low-birthweight infants with patent ductus arteriosus. Pediatr Res. 2016;80(6):785-92.

40. Weisz DE, Mirea L, Rosenberg E, Jang M, Ly L, Church PT, et al. Association of Patent Ductus Arteriosus Ligation with Death or neurodevelopmental impairment among extremely preterm infants. JAMA Pediatr. 2017;171(5): 443-9.

\section{Publisher's Note}

Springer Nature remains neutral with regard to jurisdictional claims in published maps and institutional affiliations.

Ready to submit your research? Choose BMC and benefit from:

- fast, convenient online submission

- thorough peer review by experienced researchers in your field

- rapid publication on acceptance

- support for research data, including large and complex data types

- gold Open Access which fosters wider collaboration and increased citations

- maximum visibility for your research: over $100 \mathrm{M}$ website views per year

At $\mathrm{BMC}$, research is always in progress.

Learn more biomedcentral.com/submissions 